\title{
CT perfusion during delayed cerebral ischemia after subarachnoid hemorrhage: distinction between reversible ischemia and ischemia progressing to infarction
}

\author{
Charlotte H. P. Cremers ${ }^{1,2} \cdot{\text { Pieter C. } \text { Vos }^{3} \text { • Irene C. van der Schaaf }}^{2}$ • \\ Birgitta K. Velthuis $^{2} \cdot$ Mervyn D. I. Vergouwen $^{1}$ • Gabriel J. E. Rinkel ${ }^{1}$ • \\ Jan Willem Dankbaar ${ }^{2}$
}

Received: 8 February 2015 / Accepted: 14 May 2015 /Published online: 2 June 2015

(C) The Author(s) 2015. This article is published with open access at Springerlink.com

\begin{abstract}
Introduction Delayed cerebral ischemia (DCI) after aneurysmal subarachnoid hemorrhage (aSAH) can be reversible or progress to cerebral infarction. In patients with a deterioration clinically diagnosed as DCI, we investigated whether CT perfusion (CTP) can distinguish between reversible ischemia and ischemia progressing to cerebral infarction.

Methods From a prospectively collected series of aSAH patients, we included those with DCI, CTP on the day of clinical deterioration, and follow-up imaging. In qualitative CTP analyses (visual assessment), we calculated positive and negative predictive value (PPV and NPV) with $95 \%$ confidence intervals $(95 \% \mathrm{CI})$ of a perfusion deficit for infarction on follow-up imaging. In quantitative analyses, we compared perfusion values of the least perfused brain tissue between patients with and without infarction by using receiver-operator characteristic curves and calculated a threshold value with PPV and NPV for the perfusion parameter with the highest area under the curve. Results In qualitative analyses of 33 included patients, 15 of 17 patients $(88 \%)$ with and 6 of 16 patients $(38 \%)$ without infarction on follow-up imaging had a perfusion deficit during clinical deterioration $(p=0.002)$. Presence of a perfusion deficit had a PPV of $71 \%(95 \%$ CI: $48-89 \%)$ and NPV of $83 \%$
\end{abstract}

Charlotte H. P. Cremers

C.H.P.Cremers-2@umcutrecht.nl

1 Department of Neurology and Neurosurgery, Brain Center Rudolf Magnus, University Medical Center Utrecht, PO Box 85500, 3508 GA Utrecht, Utrecht, The Netherlands

2 Department of Radiology, University Medical Center Utrecht, Utrecht, The Netherlands

3 Image Sciences Institute, University Medical Center Utrecht, Utrecht, The Netherlands
(95\%CI: 52-98 \%) for infarction on follow-up. Quantitative analyses showed that an absolute minimal cerebral blood flow (CBF) threshold of $17.7 \mathrm{~mL} / 100 \mathrm{~g} / \mathrm{min}$ had a PPV of $63 \%$ (95\%CI: $41-81 \%$ ) and a NPV of $78 \%$ (95\%CI: $40-97 \%$ ) for infarction.

Conclusions CTP may differ between patients with DCI who develop infarction and those who do not. For this purpose, qualitative evaluation may perform marginally better than quantitative evaluation.

Keywords CT perfusion · Delayed cerebral ischemia . Subarachnoid hemorrhage

\section{Introduction}

Delayed cerebral ischemia (DCI) is a severe complication of aneurysmal subarachnoid hemorrhage (aSAH). DCI affects approximately $20-30 \%$ of patients who survive the initial hemorrhage and usually occurs between 4 and 14 days after the hemorrhage $[1,2]$. Clinical deterioration from DCI can present as a new focal neurologic impairment, a decrease in the level of consciousness, or both [3]. DCI can be reversible or progress to cerebral infarction, which increases the risk of poor functional outcome [4-6]. DCI is mainly a clinical diagnosis at onset based on exclusion of other causes of the deterioration and confirmed by follow-up imaging showing cerebral infarction, but recent studies have shown that CT perfusion (CTP) can be helpful to detect DCI at the time of clinical deterioration [7]. However, it remains unknown if patients with reversible ischemia can be distinguished from patients with ischemia progressing to cerebral infarction. Such differentiation would be useful for clinical trials testing the efficacy of interventions to prevent cerebral infarction. DCI can be 
assessed with CTP in different ways: qualitatively (with visual detection of a perfusion deficit) and with quantitative thresholds (both absolute and relative).

The purpose of this study was to investigate whether CTP can distinguish between reversible ischemia and ischemia progressing to cerebral infarction.

\section{Methods}

\section{Design}

This study was approved by the institutional review board. Patients were selected from a prospectively collected series of consecutive aSAH patients admitted to our hospital between August 2007 and November 2010. In our hospital, all patients with aSAH routinely undergo multimodal CT, consisting of non-contrast CT (NCCT), CTP, and CTangiography (CTA), on admission and at the time of clinical deterioration after aSAH. Inclusion criteria for this study were (1) 18 years of age or older, (2) clinical deterioration due to DCI within 21 days after aSAH, (3) NCCT and CTP imaging at the onset of clinical deterioration, and (4) follow-up imaging (CT or MRI) at least 3 days after onset of clinical deterioration. Exclusion criteria were pregnancy, impaired renal function (creatinine $>200 \mu \mathrm{mol} / \mathrm{L}$ ) or other contraindications for contrast administration, and CTP scans with extensive movement artifacts. In our institution, none of the SAH patients are treated with endovascular rescue therapy.

\section{DCI}

Clinical deterioration due to DCI was defined as a clinical deterioration (new focal deficit or decrease in Glasgow Coma Scale of at least two points on the total score or one of its individual components or both) lasting $1 \mathrm{~h}$ or longer with no evidence for rebleeding or hydrocephalus on $\mathrm{CT}$ and no other medical causes, such as cardiovascular or pulmonary complications, infections, or metabolic disturbances [3].

Cerebral infarction due to DCI was defined as a new infarction on follow-up CT or MRI that was not present on earlier imaging and could not be attributed to other causes such as aneurysm treatment, ventricular catheter placement, or intraparenchymal hematoma [3].

\section{CTP imaging}

All imaging studies were performed on a 16-, 64-, or 128multidetector CT scanner (Philips Mx8000 IDT 16, Philips Brilliance 16P, Philips Brilliance 64, Philips Brilliance iCT; Best, the Netherlands). For the CTP scan, $40 \mathrm{ml}$ of non-ionic contrast agent (lopromide, Ultravist, $300 \mathrm{mg}$ iodine $/ \mathrm{ml}$, Schering, Berlin, Germany) was injected into the cubital vein (18-gauge needle) at a rate of $5 \mathrm{ml} / \mathrm{s}$ followed by a $40 \mathrm{ml}$ saline flush at a rate of $5 \mathrm{ml} / \mathrm{s}$ using a dual power injector (Stellant Dual CT injector, Medrad Europe BV, Beek, the Netherlands). The following parameters were used: 16 slice, $90 \mathrm{kVp}$, $150 \mathrm{mAs}, 8 \times 3 \mathrm{~mm}$ collimation; 64 slice, $80 \mathrm{kVp}, 150 \mathrm{mAs}$, $64 \times 0.625 \mathrm{~mm}$ collimation; 128 slice, $80 \mathrm{kVp}, 150 \mathrm{mAs}$, $128 \times 0.625 \mathrm{~mm}$ collimation. All scanners used a $512 \times 512$ matrix, a field of view ranging from 160 to $220 \mathrm{~mm}$, UB filter and standard resolution and acquired one image per $2 \mathrm{~s}$ during $60 \mathrm{~s}$.

\section{CTP post-processing}

CT perfusion maps were generated using an open source software package (Perfusion Mismatch Analyzer, PMA version 4.0.4.4, ASIST Japan). The optimal arterial input function (AIF) and venous output function (VOF) were automatically selected by the software and corrected manually if necessary. Patient movement was corrected with Elastix, and for noise reduction, a filter using the time-intensity profile similarity to reduce noise in the spatial domain was applied [8,9]. Quantitative cerebral perfusion values were calculated using blockcirculant singular value decomposition (bSVD), which is described as a tracer delay-insensitive algorithm [10]. CTP maps were created for cerebral blood flow (CBF), cerebral blood volume (CBV), mean transit time (MTT), and time to peak (TTP).

An in-house software tool was developed using Mevislab ( ${ }^{\mathbb{B}}$ MevisLab, software for medical image processing and visualization; http://www.mevislab.de) for visualization of the perfusion maps and to extract statistics from regions of interest (ROIs) that were manually placed on the perfusion maps.

\section{CTP evaluation}

CT perfusion maps were evaluated qualitatively (visual assessment) and quantitatively. Qualitative evaluation was done by one observer (JWD, 8-year experience with CTP in DCI) who was blinded for follow-up imaging and the final diagnosis but with knowledge of the patient's clinical condition at the time of imaging (GCS-scores and focal deficit if applicable). For every patient, the four CTP maps were displayed together with the NCCT. Positive findings were hypoperfused areas (lower CBF or CBV or higher MTT or TTP), which were not localized in the neurosurgical trajectory, directly surrounding an intracerebral hematoma, or caused by an endovascular intervention.

For quantitative analyses, we investigated the least perfused region of the brain by selecting the lowest $\mathrm{CBF}$ and CBV value and the highest MTT and TTP value. The least perfused region was selected from standard ROIs and perfusion deficits (if applicable): 
1. Standard ROIs were drawn in the cortical flow territories of the anterior cerebral artery (ACA), middle cerebral artery (MCA), and posterior cerebral artery (PCA) and in the basal ganglia (Fig. 1). Because perfusion in unaffected white matter can be lower than perfusion in a perfusion deficit in gray matter, we did not include white matter regions in the standard ROIs.

2. Perfusion deficits. In all patients with a visible perfusion deficit on CTP during clinical deterioration, the deficit was manually delineated. Delineation was done blinded for the results of follow-up imaging.

For relative perfusion values, the least perfused ROI was compared to the contralateral ROI. Subsequently, for CBF and CBV minimal ratios of cerebral perfusion values were calculated and for MTT and TTP maximal differences of perfusion values were calculated.

\section{Analyses}

For the qualitative analyses, we calculated positive and negative predictive values (PPV and NPV) with corresponding $95 \%$ confidence intervals $(95 \% \mathrm{CI})$ of presence of perfusion deficits for infarction on follow-up. Subsequently, we determined the proportion of patients with a perfusion deficit on CTP during clinical deterioration for patients with and without an infarction on follow-up imaging. Differences between groups were analyzed using the chi-square test.

For the quantitative analyses, we constructed a receiveroperator characteristic (ROC) curve for the distinction between patients with and without an infarction on follow-up imaging for all parameters and calculated the area under the curve (AUC). Of all perfusion parameters, the parameter with the highest AUC was selected and, subsequently, PPV and NPV (with $95 \%$ CI) for the threshold value with the optimal diagnostic cutoff point were calculated.

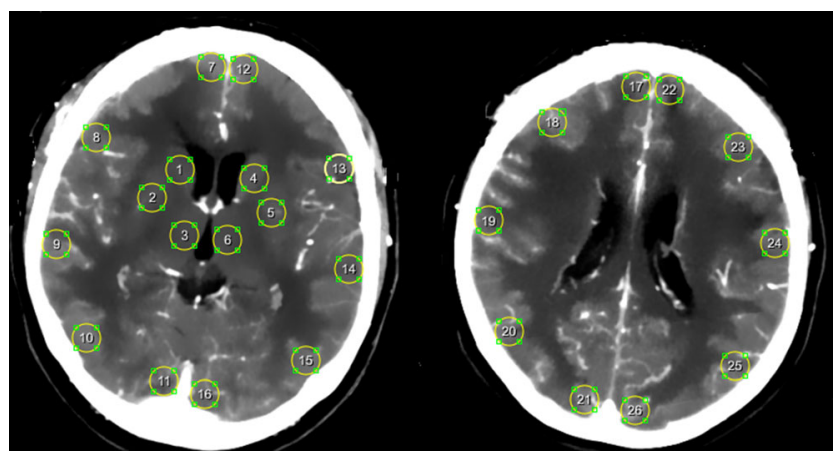

Fig. 1 Standard ROIs in the cortical flow territories of the anterior cerebral artery, middle cerebral artery, and posterior cerebral artery and in the basal ganglia

\section{Results}

We included 33 patients with clinical deterioration due to DCI, of which 17 patients had an infarction on follow-up imaging (Table 1). Four patients were scanned on a 16-multidetector CT scanner, 18 patients on a 64-multidetector CT scanner, and 11 patients on a 128 -multidetector CT scanner.

\section{Qualitative analyses}

A perfusion deficit was seen on CTP during clinical deterioration in 15 of 17 patients ( $88 \%$ ) with a final infarction on follow-up imaging compared to 6 of 16 patients ( $38 \%$ ) without a final infarction $(p=0.002)$ (Table 1$)$. The PPV for the development of an infarction was $71 \%$ (95\%CI: 48-89\%) and the NPV was $83 \%$ (95\%CI: 52-98 \%).

\section{Quantitative analyses}

For the least perfused region of the brain, median perfusion values (with interquartile ranges (IQR)) for DCI patients with and without an infarction on follow-up are presented in Table 2. A trend towards lower CBF and CBV and longer MTT and TTP was seen in patients with infarction compared to patients without an infarction on follow-up imaging. The AUC of the ROC curve for absolute CBF was 0.65 , for CBV 0.58, for MTT 0.60, and for TTP 0.50. For the relative perfusion values, the AUCs were for CBF 0.52 , CBV 0.47, MTT 0.57, and for TTP 0.52. Since the absolute $\mathrm{CBF}$ had the highest AUC, this parameter was selected for further analysis. An absolute CBF threshold of $17.7 \mathrm{~mL} / 100 \mathrm{~g} / \mathrm{min}$ showed a PPV of $63 \%$ (95\%CI: $41-$ $81 \%$ ) and a NPV of $78 \%$ (95\%CI: $40-97 \%$ ).

\section{Discussion}

The results of our study show that in patients with clinical deterioration due to DCI, qualitative CTP assessment predicts better an infarction on follow-up than quantitative assessment; however, the predictive values of perfusion deficits are only moderate. Patients with a visually assessed perfusion deficit at the time of clinical deterioration more often have an infarction on follow-up imaging than such patients without a perfusion deficit.

The distinction between reversible ischemia and ischemia progressing to infarction in patients with clinical deterioration due to DCI has been studied only once before in a subgroup analysis of a study investigating the value of CTP in predicting outcome after aSAH [11]. In that study, perfusion scans were visually assessed and additionally quantitative measurements were performed in standard ROIs. In contrast to our results, that study found no significant difference in the 
Table 1 Patient characteristics

\begin{tabular}{|c|c|c|}
\hline & $\begin{array}{l}\text { DCI with } \\
\text { infarction } \\
(n=17)\end{array}$ & $\begin{array}{l}\text { DCI without } \\
\text { infarction } \\
(n=16)\end{array}$ \\
\hline Women (n, \%) & $12(71 \%)$ & $14(88 \%)$ \\
\hline Age (mean, range) & $52(29-67)$ & $58(28-81)$ \\
\hline \multicolumn{3}{|l|}{ Admission WFNS score (\%) } \\
\hline I & $7(41 \%)$ & $4(25 \%)$ \\
\hline II & $5(29 \%)$ & $5(31 \%)$ \\
\hline III & $2(12 \%)$ & $1(6 \%)$ \\
\hline IV & $1(6 \%)$ & $4(25 \%)$ \\
\hline V & $2(12 \%)$ & $2(13 \%)$ \\
\hline \multicolumn{3}{|l|}{ Aneurysm location (\%) } \\
\hline Acom, ACA & $8(47 \%)$ & $7(44 \%)$ \\
\hline Pcom & $3(18 \%)$ & $5(31 \%)$ \\
\hline BA & $3(18 \%)$ & $2(13 \%)$ \\
\hline ICA & $3(18 \%)$ & $2(13 \%)$ \\
\hline \multicolumn{3}{|l|}{ Treatment $(\%)$} \\
\hline Coil & $10(59 \%)$ & $9(56 \%)$ \\
\hline Clip & $6(35 \%)$ & $6(38 \%)$ \\
\hline None & $0(0 \%)$ & $1(6 \%)$ \\
\hline Both & $1(6 \%)$ & $0(0 \%)$ \\
\hline Hypertension induction as treatment for DCI (\%) & $5(29 \%)$ & $4(25 \%)$ \\
\hline Perfusion deficit visible on CTP during clinical deterioration (\%) & $15(88 \%)$ & $6(38 \%)$ \\
\hline \multicolumn{3}{|l|}{ Clinical presentation at time of clinical deterioration (\%) } \\
\hline \multicolumn{3}{|l|}{ WFNS score } \\
\hline I & $0(0 \%)$ & $0(0 \%)$ \\
\hline II & $1(6 \%)$ & $1(6 \%)$ \\
\hline III & $1(6 \%)$ & $1(6 \%)$ \\
\hline IV & $6(35 \%)$ & $3(19 \%)$ \\
\hline $\mathrm{V}$ & $2(12 \%)$ & $5(31 \%)$ \\
\hline Focal deficit & $6(35 \%)$ & $5(31 \%)$ \\
\hline Unknown & $1(6 \%)$ & $1(6 \%)$ \\
\hline \multicolumn{3}{|c|}{ Mean CTP value (with SD) per patient (standard ROIs and perfusion deficits) } \\
\hline $\mathrm{CBF}(\mathrm{mL} / 100 \mathrm{~g} / \mathrm{min})$ & $25.29(9.76)$ & $30.38(12.43)$ \\
\hline CBV (mL/100 g) & $2.79(0.98)$ & $3.21(1.15)$ \\
\hline MTT (s) & $14.43(1.97)$ & $13.88(1.07)$ \\
\hline TTP (s) & $14.20(3.04)$ & $14.09(2.09)$ \\
\hline
\end{tabular}

$D C I$ delayed cerebral ischemia, WFNS World Federation of Neurosurgeons, Acom anterior communicating artery, $A C A$ anterior cerebral artery, $P$ com posterior communicating artery, $B A$ basilar artery, $I C A$ internal carotid artery, CTP CT perfusion, $S D$ standard deviation, ROIs regions of interest, $C B F$ cerebral blood flow, $C B V$ cerebral blood volume, MTT mean transit time, TTP time to peak

qualitative analyses of patients with and without an infarction, although a trend was seen to a higher proportion of visually assessed CTP deficits in DCI patients with infarction on follow-up compared to DCI patients without infarction [11]. In that study, 15 patients with infarction and 33 patients without infarction were included. Different software packages were used in both studies to generate the perfusion maps, which might explain the different results that were found. Therefore, standardized methods for measuring perfusion with CTP after SAH are needed [7]. In the quantitative analyses of that study also no significant differences were found between DCI patients with and without an infarction. Our study design for the quantitative analyses differs essentially from the previous study. We measured perfusion in the area with the lowest perfusion in each patient in a visually delineated perfusion deficit or in the absence of a perfusion deficit in the lowest standard ROI. In contrast, the previous study measured perfusion either in 
Table 2 Lowest perfusion values

\begin{tabular}{lll}
\hline CTP parameter & Infarction at follow-up $(n=17)$ & No infarction at follow-up $(n=16)$ \\
\hline Absolute values (median (IQR)) & & \\
$\quad$ CBF $(\mathrm{mL} / 100 \mathrm{~g} / \mathrm{min})$ & $11.66(8.34-16.32)$ & $16.08(8.92-23.57)$ \\
CBV $(\mathrm{mL} / 100 \mathrm{~g})$ & $1.66(1.07-2.14)$ & $1.78(1.23-2.44)$ \\
MTT $(\mathrm{s})$ & $21.72(16.76-26.54)$ & $18.83(15.65-23.05)$ \\
TTP $(\mathrm{s})$ & $19.18(14.86-23.76)$ & $18.24(16.29-21.94)$ \\
Relative values (median $(\mathrm{IQR}))$ & & $0.56(0.36-0.62)$ \\
CBF ratio & $0.53(0.42-0.60)$ & $0.59(0.45-0.65)$ \\
CBV ratio & $0.57(0.53-0.65)$ & $4.68(2.29-9.44)$ \\
MTT difference $(\mathrm{s})$ & $7.62(2.96-12.10)$ & $4.46(1.77-8.01)$ \\
TTP difference $(\mathrm{s})$ & $3.77(2.16-8.92)$ &
\end{tabular}

Lowest perfusion values: lowest value and ratio in $\mathrm{CBF}$ and $\mathrm{CBV}$ and highest value and difference in MTT and TTP, measured in standard ROIs in gray matter and basal ganglia or perfusion deficits (if applicable)

$C T P$ CT perfusion, $n$ number, IQR interquartile range (25-75\%), $C B F$ cerebral blood flow, $C B V$ cerebral blood volume, MTT mean transit time, TTP time to peak.

standard ROIs located within a perfusion deficit or used, in those patients without a perfusion deficit, the mean value of all standard ROIs instead of the ROI with lowest perfusion [11]. In our opinion, the comparison of the measurements in a perfusion deficit to the lowest values in patients without deficits is more realistic if quantitative differences between the two groups are being evaluated.

The absolute perfusion values found in our study are lower compared to perfusion values found in other studies in DCI patients $[12,13]$. This can be explained by the fact that we measured perfusion in perfusion deficits and used the least perfused region, whereas in other studies the mean value of standard ROIs was used. In addition, quantitative perfusion values may differ due to differences in CTP algorithms [14]. Because absolute perfusion values are known to vary and may be influenced by user-dependent post-processing steps [14, 15], we also evaluated relative CTP measurements and found comparable results to the absolute CTP measurements.

In patients with ischemic stroke, a prediction map based on quantitative thresholds is used to distinguish between reversible and irreversible ischemic tissue [16]. Since we found lower predictive values for the quantitative thresholds compared to the qualitative assessment in this study, the development of a prediction map in DCI patients seems less useful than visual assessment.

Although the PPV and NPV of the visual assessment with CTP found in this study were not overwhelming, the visual assessment of CTP could be useful in clinical practice to give an indication of the chance of developing an infarction due to DCI. However, the predictive values of the visual assessment of CTP to distinguish between DCI patients with and without infarction need to be validated in a prospective study. In addition, its value for predicting outcome needs to be evaluated.
Other techniques like diffusion-weighted MRI may be more suited to detect irreversible ischemia in an early stage [17]. However, the use of MRI is often challenging in SAH patients because of incompatibility of monitoring equipment with the MR suite. In addition, in many hospitals, MRI is still not available around the clock. Several technical improvements for CTP imaging are currently being investigated [18-20]. This may make more accurate estimation of irreversible ischemia possible in the future.

Some limitations need to be addressed. Although the definition for DCI used in our study is widely accepted [3], the diagnosis of DCI is based on excluding other causes of clinical deterioration. Since there are many factors influencing the occurrence of clinical deterioration after aSAH, there may still be some misclassification, which may influence predictive values. Another potential limitation is that the qualitative assessment of the CTP scans was done by a single observer. In a previous study, lower test characteristics were found for a less experienced observer compared to an experienced observer; however this was based on only two observers [21]. In stroke studies, good reproducibility was found for visual assessment of CTP images [22]. Finally, although the percentage of patients receiving induced hypertension was similar in patients with and without an infarct on follow-up, the change in blood pressure and thereby cerebral blood flow may not have been equal. This may have introduced some bias. On the other hand, there are no data available in the literature on the effect of induced hypertension on the development of infarction.

To conclude, CTP may differ between patients with DCI who develop infarction and those who do not, but before these findings can be implemented in clinical practice, they should be confirmed in different patient 
populations in different centers. To differentiate between the two groups, qualitative analysis may perform marginally better than quantitative analysis.

Ethical standards and patient consent We declare that all human and animal studies have been approved by the appropriate Ethics Committee and have therefore been performed in accordance with the ethical standards laid down in the 1964 Declaration of Helsinki and its later amendments. We declare that all patients gave informed consent prior to inclusion in this study.

Conflict of interest We declare that we have no conflict of interest.

Open Access This article is distributed under the terms of the Creative Commons Attribution 4.0 International License (http:// creativecommons.org/licenses/by/4.0/), which permits unrestricted use, distribution, and reproduction in any medium, provided you give appropriate credit to the original author(s) and the source, provide a link to the Creative Commons license, and indicate if changes were made.

\section{References}

1. Roos YB, de Haan RJ, Beenen LF, Groen RJ, Albrecht KW, Vermeulen M (2000) Complications and outcome in patients with aneurysmal subarachnoid haemorrhage: a prospective hospital based cohort study in the Netherlands. J Neurol Neurosurg Psychiatry 68:337-341

2. Dorhout Mees SM, Kerr RS, Rinkel GJ, Algra A, Molyneux AJ (2012) Occurrence and impact of delayed cerebral ischemia after coiling and after clipping in the International Subarachnoid Aneurysm Trial (ISAT). J Neurol 259:679-683

3. Vergouwen MD, Vermeulen M, Van Gijn J, Rinkel GJ, Wijdicks EF, Muizelaar JP et al (2010) Definition of delayed cerebral ischemia after aneurysmal subarachnoid hemorrhage as an outcome event in clinical trials and observational studies: proposal of a multidisciplinary research group. Stroke 41:2391-2395

4. Vergouwen MD, Etminan N, Ilodigwe D, Macdonald RL (2011) Lower incidence of cerebral infarction correlates with improved functional outcome after aneurysmal subarachnoid hemorrhage. J Cereb Blood Flow Metab 31:1545-1553

5. Vergouwen MD, Ilodigwe D, Macdonald RL (2011) Cerebral infarction after subarachnoid hemorrhage contributes to poor outcome by vasospasm-dependent and -independent effects. Stroke 42:924-929

6. Dankbaar JW, de Rooij NK, Smit EJ, Velthuis BK, Frijns CJ, Rinkel GJ et al (2011) Changes in cerebral perfusion around the time of delayed cerebral ischemia in subarachnoid hemorrhage patients. Cerebrovasc Dis 32:133-140

7. Cremers CH, van der Schaaf IC, Wensink E, Greving JP, Rinkel GJ, Velthuis BK et al (2014) CT perfusion and delayed cerebral ischemia in aneurysmal subarachnoid hemorrhage: a systematic review and meta-analysis. J Cereb Blood Flow Metab 34:200-207

8. Klein S, Staring M, Murphy K, Viergever MA, Pluim JP (2010) Elastix: a toolbox for intensity-based medical image registration. IEEE Trans Med Imaging 29:196-205

9. Mendrik AM, Vonken EJ, van Ginneken B, de Jong HW, Riordan A, van Seeters T et al (2011) TIPS bilateral noise reduction in 4D
CT perfusion scans produces high-quality cerebral blood flow maps. Phys Med Biol 56:3857-3872

10. Wu O, Ostergaard L, Weisskoff RM, Benner T, Rosen BR, Sorensen AG (2003) Tracer arrival timing-insensitive technique for estimating flow in MR perfusion-weighted imaging using singular value decomposition with a block-circulant deconvolution matrix. Magn Reson Med 50:164-174

11. Sanelli PC, Anumula N, Johnson CE, Comunale JP, Tsiouris AJ, Riina $\mathrm{H}$ et al (2013) Evaluating CT perfusion using outcome measures of delayed cerebral ischemia in aneurysmal subarachnoid hemorrhage. AJNR Am J Neuroradiol 34:292-298

12. Chai WN, Sun XC, Lv FJ, Wan B, Jiang L (2011) Clinical study of changes of cerebral microcirculation in cerebral vasospasm after SAH. Acta Neurochir Suppl 110:225-228

13. Killeen RP, Mushlin AI, Johnson CE, Comunale JP, Tsiouris AJ, Delaney $\mathrm{H}$ et al (2011) Comparison of CT perfusion and digital subtraction angiography in the evaluation of delayed cerebral ischemia. Acad Radiol 18:1094-1100

14. Abels B, Villablanca JP, Tomandl BF, Uder M, Lell MM (2012) Acute stroke: a comparison of different CT perfusion algorithms and validation of ischaemic lesions by follow-up imaging. Eur Radiol 22:2559-2567

15. Kudo K, Sasaki M, Yamada K, Momoshima S, Utsunomiya H, Shirato H et al (2010) Differences in CT perfusion maps generated by different commercial software: quantitative analysis by using identical source data of acute stroke patients. Radiology 254:200209

16. Wintermark M, Flanders AE, Velthuis B, Meuli R, van Leeuwen M, Goldsher D et al (2006) Perfusion-CT assessment of infarct core and penumbra: receiver operating characteristic curve analysis in 130 patients suspected of acute hemispheric stroke. Stroke 37:979 985

17. Sorensen AG, Buonanno FS, Gonzalez RG, Schwamm LH, Lev MH, Huang-Hellinger FR et al (1996) Hyperacute stroke: evaluation with combined multisection diffusion-weighted and hemodynamically weighted echo-planar MR imaging. Radiology 199:391401

18. Riordan AJ, Bennink E, Viergever MA, Velthuis BK, Dankbaar JW, de Jong HW (2013) CT brain perfusion protocol to eliminate the need for selecting a venous output function. AJNR Am J Neuroradiol 34:1353-1358

19. Bennink E, Riordan AJ, Horsch AD, Dankbaar JW, Velthuis BK, de Jong HW (2013) A fast nonlinear regression method for estimating permeability in CT perfusion imaging. J Cereb Blood Flow Metab 33:1743-1751

20. Dolatowski K, Malinova V, Frolich AM, Schramm R, Haberland U, Klotz E et al (2014) Volume perfusion CT (VPCT) for the differential diagnosis of patients with suspected cerebral vasospasm: qualitative and quantitative analysis of 3D parameter maps. Eur J Radiol 83:1881-1889

21. Cremers CH, Dankbaar JW, Vergouwen MD, Vos PC, Bennink E, Rinkel GJ, Velthuis BK, van der Schaaf IC. Different CT perfusion algorithms in the detection of delayed cerebral ischemia after aneurysmal subarachnoid hemorrhage. Neuroradiology. Accepted for publication

22. van Seeters T, Biessels GJ, Niesten JM, van der Schaaf IC, Dankbaar JW, Horsch AD et al (2013) Reliability of visual assessment of non-contrast CT, CT angiography source images and CT perfusion in patients with suspected ischemic stroke. PLoS One 8: e75615 